Marissa Rase*, Mark Hanlon, Le Ho, Doug Duriez and Cathy Zhao

\title{
Vial coring and fragmentation incidence after angled penetration of rubber stoppers with single-use hypodermic needles
}

https://doi.org/10.1515/pthp-2021-0004

Received May 5, 2021; accepted July 28, 2021;

published online September 1, 2021

\section{Abstract}

Objectives: Particles due to fragmentation present a clear risk to the patient. Reported fragmentation rates vary, and an insertion angle at $45^{\circ}$, as opposed to $90^{\circ}$, has been proposed as a mitigation strategy. So, this study evaluated the fragmentation rates induced by single-use hypodermic needles using different angled penetration techniques.

Methods: Needles underwent fragmentation testing using two penetration techniques. In method 1 , the needle was inserted through the stopper at $45^{\circ}$ and rotated to $90^{\circ}$ upon exiting the stopper underside, and in method 2 the needle was rotated only after the bevel was fully enveloped by the stopper. Methods were tested with 18, 20, and 22-gauge needles with bevel faced up, down, and sideways. Fragmentation data sets were subjected to ANOVA and a fit to a General Linear Model was attempted to ascertain the significance of needle size, bevel position, and penetration method; p-values less than 0.05 indicated statistical significance.

Results: Incidence varied from 0 to $49 \%$ and depended on the test method. Needles larger than 22-gauge induced fragmentation the most when the bevel was down. The bevel up position induced fragmentation the least. Generation of large fragments designated "cores" depended on all factors examined, and generation of small fragments designated "fragments" depended on all factors except for the penetration method.

\footnotetext{
*Corresponding author: Marissa Rase, Scientific Insights, West Pharmaceutical Services, Inc., 530 Herman O. West Drive, Exton, PA 19341, USA, Phone: (610) 906 6837,

E-mail: Marissa.Rase@westpharma.com

Mark Hanlon, Technical Operations, Endo Pharmaceuticals, Dublin, Ireland

Le Ho and Cathy Zhao, Scientific Insights, West Pharmaceutical Services, Inc., Exton, PA, USA

Doug Duriez, R\&D, Formulation Development, West Pharmaceutical Services, Inc., Exton, PA, USA
}

Conclusions: Clinical context and intended application need to be communicated to manufacturers and considered for functional testing when devising end-user recommendations which must reflect a combination of factors.

Keywords: container closure systems; coring; fragmentation; parenteral drug products; patient safety; rubber stoppers.

\section{Introduction}

A very common form of medication delivery in the clinical setting involves its withdrawal from sealed stopper-vial systems into syringes for injection. This mode of vial access is of interest and point of concern for clinical units where it is commonplace, such as within pharmacy chemotherapy preparation units, intravenous additive services, and intensive care settings. For example, a hospital reported that approximately 99,000 rubber stoppers from various medication vials were pierced in its intensive care unit in 2015 [1]. It is thusly paramount to patient care to understand the limitations of this widely used system. For any given needle passing through a rubber stopper there is the risk of abrasion, tearing, and cutting which results in rubber particles entering the medication vial or being retained in the needle or cannula during events known as fragmentation. During insertion the needle bevel heel can also scoop out large fragments from the stopper in fragmentation events known as coring. Sources vary on the estimated frequency rate of fragmentation wherein some cite it as a low frequency event [2-4] while others cite it as a common, yet underreported, event in the clinical setting $[5,6]$. Concern has been raised over these fragments as it pertains to device performance and component attributes such as with lowered elastomeric quality in the bung or stopper. Decreased resealability and tensile strength, for example, have been implicated in increasing fragmentation $[7,8]$.

Any particle entering a medication vial poses a certain level of risk to the patient that is still not well-understood. Although there are few examples in the literature, 
instances of rubber particles presenting risk in the clinic exist. For example, rubber fragments have presented potential risks for embolization during the reconstitution of BOTOX $^{\circledR}$ (onabotulinumtoxinA) products [9]. Rubber fragments, along with other microscopic particles, have also been observed in all aspirate specimens used during eye surgery which, if injected into the retrobulbar and peribulbar space, could increase inflammation and result in serious complications [10]. In another case, a rubber particle was observed occluding anesthetic devices and consequently required emergency angiocath implementation to avoid a potentially fatal embolism [11]. In the latter particularly harrowing case, the patient was only 16 months old and the event was enough justification for the healthcare provider to view fragmentation as an often underreported and very dangerous clinical event. Consequently, the provider decided to change their usual needlestick safety and purchasing procedures from blunt cannulae. Resolving the presence of these fragments, either by prevention or removal, may improve patient care.

Many basic science studies have attempted to understand and lower fragmentation incidence. Several studies have determined varying fragmentation rates, having observed anywhere between 0 and $97 \%$ fragmentation [1, $2,12,13]$. Several variables affecting fragmentation have been identified, such as stopper material, stopper and vial surface area, diaphragm thickness, improper use and application of devices and components, elastomeric properties, stopper fit to vial, needle design and material, needle point sharpness, medication fill volume, penetration angle, and the type of medication contained in the vial $[7,12]$. These factors create an impractical testing burden, which places a strain on manufacturers and regulatory bodies in validating the devices produced by manufacturers. Commonly suggested alternative solutions include filter needles and pre-filled syringes; however, several medications are not stable in solution or are too viscous to be applied in pre-filled syringes or through filter needles [14]. Also, with the advent of closed transfer devices in clinics administering hazardous drugs, which have demonstrated an unresolved fragmentation problem, these safety accessories are not always compatible $[15,16]$. Some have suggested removing the stopper altogether, but this creates obvious concerns over contamination [17].

A very direct mode of mitigating fragmentation risk in the clinic is to evaluate the current technique for medication withdrawal as it pertains to fragmentation, and to make changes in the practice accordingly. It is the general consensus among healthcare providers that it is the manufacturer's responsibility to recommend best-practices for their device that mitigate fragmentation and the healthcare provider's responsibility to be aware of and adhere to the instructions for use [17]. It is also the opinion of regulatory agencies that the quality of a device or component is directly related to its particle formation $[18,19]$. Therefore, it is the responsibility of the manufacturer to ensure that their parenteral preparations do not contain visible particulates or are essentially free of particulates and other readily detectable insoluble material [18-25]. Recent proposed changes to the United States Pharmacopeia also reflect the necessity to account for fragmentation within the context of a device's intended end-use [20, 21]. Thus, this study aimed to identify the effects of needle size and bevel orientation relative to the stopper surface on fragmentation incidence, as well as to identify the more appropriate penetration techniques for end-users to consider. It is also the aim for this study to illustrate a fitness-for-intended-use fragmentation test that may be performed, in addition to other appropriate functional tests covered by relevant pharmacopeias, to evaluate the suitability of a 45-degree stoppered-vial penetration technique using sterile, single-use hypodermic needles.

\section{Materials and methods}

Commercially available products used are listed in Table 1. Manufacturer names of sampled needles have been omitted. The 18-gauge (18G), 22G, and $30 \mathrm{G}$ needles were procured from the manufacturer designated "Manufacturer A." Due to product unavailability at time of testing from Manufacturer A, the $20 \mathrm{G}$ needles were instead purchased from a secondary manufacturer designated "Manufacturer B." All needles were single-use stainless steel conventional hypodermic needles equipped with a lancet point regular bevel. All stoppers, $20 \mathrm{~mm}$ film-coated bromobutyl formulation, were steam sterilized by autoclaving at $121^{\circ} \mathrm{C}$ for $1 \mathrm{~h}$ before use. A fragmentation event for this study was defined as the generation of rubber particles in the medication vial after penetration of a rubber stopper.

A protocol for fragmentation testing was adapted from current international standard methods [20-22]. As such, sterile vials and stoppers were crimp sealed under 30 psi using a jaw-style air crimper and prepared for penetration by swabbing the surface with USP grade $70 \%$ (v/v) isopropyl alcohol and allowed to dry. A $30 \mathrm{G}$ needle was used to vent the vial during testing. Needles were inserted into stoppers once using one of the test methods described in Table 2. It has been demonstrated by others that fully penetrating with the needle at $45-60^{\circ}$ followed by gradual rotation of the needle until perpendicular to the stopper surface will minimize coring $[1,3,14,26,27]$. To test this assertion, two adapted penetration techniques were examined. Penetration method 1 involved inserting the needle into the stopper target area at an angle of $45^{\circ}$ and applying pressure along this angle until the bevel point exited the underside of the diaphragm (Figure 1, Top A), the needle was then rotated slowly until perpendicular to the stopper surface and brought toward the bottom of the vial. Meanwhile, penetration method 2 involved angling the needle to $45^{\circ}$ and applying a downward pressure perpendicular to the stopper surface until the 
Table 1: Sample materials for testing.

\begin{tabular}{|c|c|c|}
\hline Material & Provider & Description \\
\hline Stopper & $\begin{array}{l}\text { West Pharmaceu- } \\
\text { tical Services }\end{array}$ & $\begin{array}{l}20 \mathrm{~mm} \text { grey bromobutyl film-coated } \\
\text { Westar }{ }^{\circledR} \text { ready-to-sterilize serum } \\
\text { stopper }\end{array}$ \\
\hline Vial & Stevanato Group & $\begin{array}{l}\text { Vial VB } 10 \mathrm{~mL} 24.00 \times 1.00 \times 45.00 \\
\text { ASBB }\end{array}$ \\
\hline Seal & $\begin{array}{l}\text { West Pharmaceu- } \\
\text { tical Services }\end{array}$ & $\begin{array}{l}20-I \text { LQ aluminum inner unlined } \\
\text { seals }\end{array}$ \\
\hline $\begin{array}{l}18 G \\
\text { Needle }\end{array}$ & Manufacturer A & $\begin{array}{l}18 \mathrm{G} \times 1^{\prime \prime} \text { sharp sterile steel, non- } \\
\text { pyrogenic, latex-free, single-use } \\
\text { hypodermic needles }\end{array}$ \\
\hline $\begin{array}{l}20 \mathrm{G} \\
\text { Needle }\end{array}$ & Manufacturer B & $\begin{array}{l}20 \mathrm{G} \times 1^{\prime \prime} \text { sharp sterile steel, non- } \\
\text { pyrogenic, latex-free, single-use } \\
\text { hypodermic needles }\end{array}$ \\
\hline $\begin{array}{l}22 \mathrm{G} \\
\text { Needle }\end{array}$ & Manufacturer A & $\begin{array}{l}22 \mathrm{G} \times 1 \text { " sharp sterile steel, non- } \\
\text { pyrogenic, latex-free, single-use } \\
\text { hypodermic needles }\end{array}$ \\
\hline $\begin{array}{l}30 \mathrm{G} \\
\text { Needle }\end{array}$ & Manufacturer A & $\begin{array}{l}30 \mathrm{G} \times 1 / 2^{\prime \prime} \text { sharp sterile steel, non- } \\
\text { pyrogenic, latex-free, single-use } \\
\text { hypodermic needles }\end{array}$ \\
\hline Filters & $\begin{array}{l}\text { GE Healthcare Life } \\
\text { Sciences }\end{array}$ & $\begin{array}{l}\text { Whatman }{ }^{\mathrm{TM}} \text { filter papers } 4 \text { qualita- } \\
\text { tive circles, diameter } 125 \mathrm{~mm} \text {, pore } \\
\text { size } 20 \mu \mathrm{m}\end{array}$ \\
\hline Syringe & $\begin{array}{l}\text { Global Medical } \\
\text { Products }\end{array}$ & $\begin{array}{l}20 \mathrm{~mL} \text { disposable easy glide luer } \\
\text { lock syringe }\end{array}$ \\
\hline
\end{tabular}

bevel was fully enveloped by the stopper (Figure 1, Top B), then the needle was rotated slowly until perpendicular to the stopper surface and pushed fully through the diaphragm toward the bottom of the vial. Each penetration method was tested for needles sized 18G, 20G, and $22 \mathrm{G}$, and at bevel orientations designated up, down, and side (Figure 1, Bottom).

Using a $20 \mathrm{~mL}$ disposable syringe, $5 \mathrm{~mL}$ of lab grade particle-free water was pumped into the vial through the needle to flush any potential fragments from the needle into the vial. The vial was gently shaken to capture any particles in the injectate, disassembled by manual de-crimping, and the contents filtered through $20 \mu \mathrm{m}$ filter paper. The filters were examined at no more than a 10 -inch distance with a $2 \times$ magnifying glass, and any filters exhibiting particulates were examined further for classification using a Nikon SMZ1000 Stereoscopic Zoom Microscope System with a 10× eyepiece and $1 \times$ objective lens equipped with a Nikon DS-U3 camera control unit and Nikon Digital Sight DS-fil digital camera. Grey particles greater than $20 \mu \mathrm{m}$ that were mobile and not affixed to or embedded in the filter paper were counted. Large cylindrical or longitudinal fragments were classified as cores (Figure 2A) and characteristically small, irregularly shaped fragments were classified only as fragments (Figure 2B). Images of the filters and stopper undersides corresponding to fragment and core presence were captured using the Nikon NIS Elements D. This protocol was performed over 100 replicates per test method for a total of 1,800 samples.

Multi-factor Analysis of Variance (ANOVA) was used to analyze the impact of each factor presented in this study on fragmentation and coring incidence. The data was fit to a General Linear Model by condensing the data from the 100 samples tested at each combination of factors into four replicate sample sets of 25 samples each using the composite number of cores and fragments for each sample set as the result for the analysis. A p-value less than 0.05 indicated statistical significance. Due to the unbalanced nature of the manufacturer covariant, the needle manufacturer was excluded from analysis.

\section{Results and discussion}

The results of testing, as summarized in Table 3, demonstrate that the needle penetration method, bevel position during penetration, manufacturer, and size all impact coring and fragmentation to various extents. There are three notable trends in fragmentation behavior due to these variables: (1) for needles larger than $22 \mathrm{G}$, the bevel down position performs the worst regardless of size, penetration method, and manufacturer; (2) The bevel up position performs most reliably for all needle sizes and bevel orientations either by fragmenting the least or not at all; (3) the dominating factor for penetration method 2 appears to be the bevel position. Stoppers that generated cores and fragments also exhibited more damage to their surface. As shown in representative images of nonfragmenting (Figure 3A) and fragmenting (Figure 3B)

Table 2: Testing method combinations.

\begin{tabular}{|c|c|c|c|c|c|c|c|}
\hline Test method & Penetration & Needle size & Bevel orientation & Test method & Penetration & Needle size & Bevel orientation \\
\hline 1 & 1 & $18 \mathrm{G}$ & Up & 10 & 2 & $18 \mathrm{G}$ & Up \\
\hline 2 & 1 & $18 \mathrm{G}$ & Down & 11 & 2 & $18 \mathrm{G}$ & Down \\
\hline 3 & 1 & $18 \mathrm{G}$ & Side & 12 & 2 & $18 \mathrm{G}$ & Side \\
\hline 4 & 1 & $20 \mathrm{G}$ & Up & 13 & 2 & $20 G$ & Up \\
\hline 5 & 1 & $20 \mathrm{G}$ & Down & 14 & 2 & $20 \mathrm{G}$ & Down \\
\hline 6 & 1 & $20 \mathrm{G}$ & Side & 15 & 2 & $20 \mathrm{G}$ & Side \\
\hline 7 & 1 & $22 \mathrm{G}$ & Up & 16 & 2 & $22 \mathrm{G}$ & Up \\
\hline 8 & 1 & $22 \mathrm{G}$ & Down & 17 & 2 & $22 \mathrm{G}$ & Down \\
\hline 9 & 1 & $22 \mathrm{G}$ & Side & 18 & 2 & $22 \mathrm{G}$ & Side \\
\hline
\end{tabular}



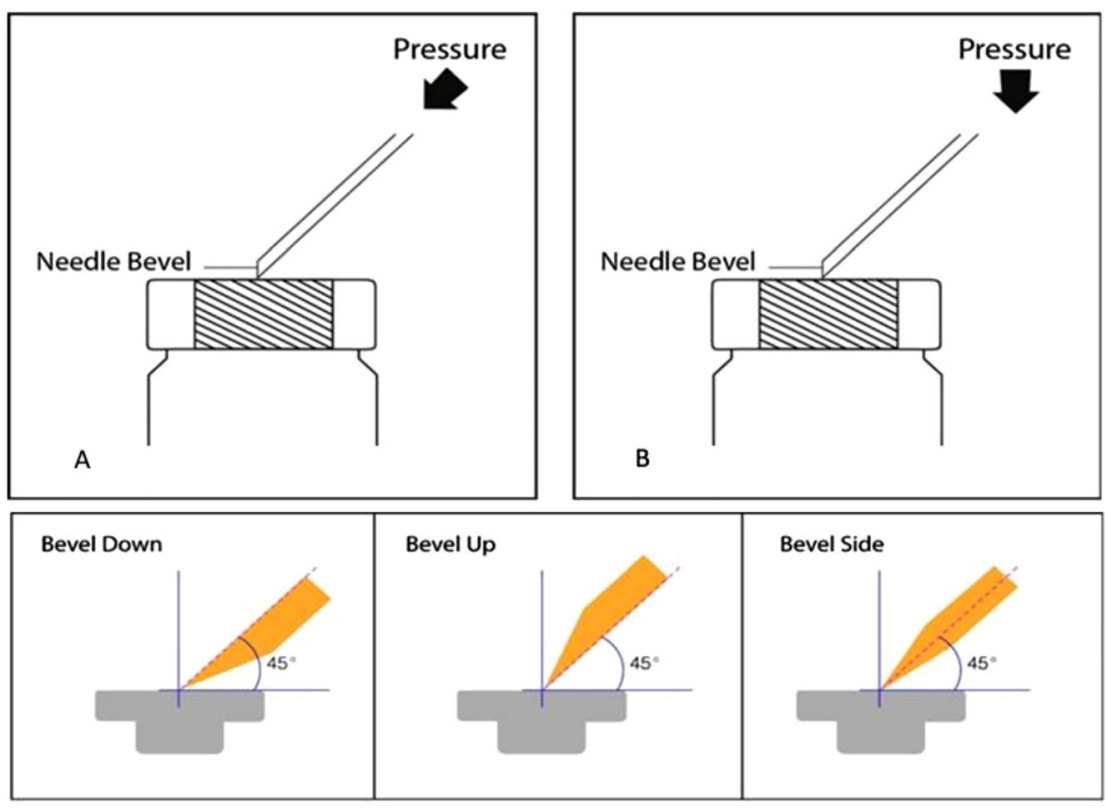

Figure 1: (Top, A) penetration method 1, angled needle entry fully through stopper diaphragm; (Top, B) penetration method 2, angled entry coupled with downward pressure until bevel fully enveloped by stopper; (Bottom) bevel positions relative to the stopper surface.
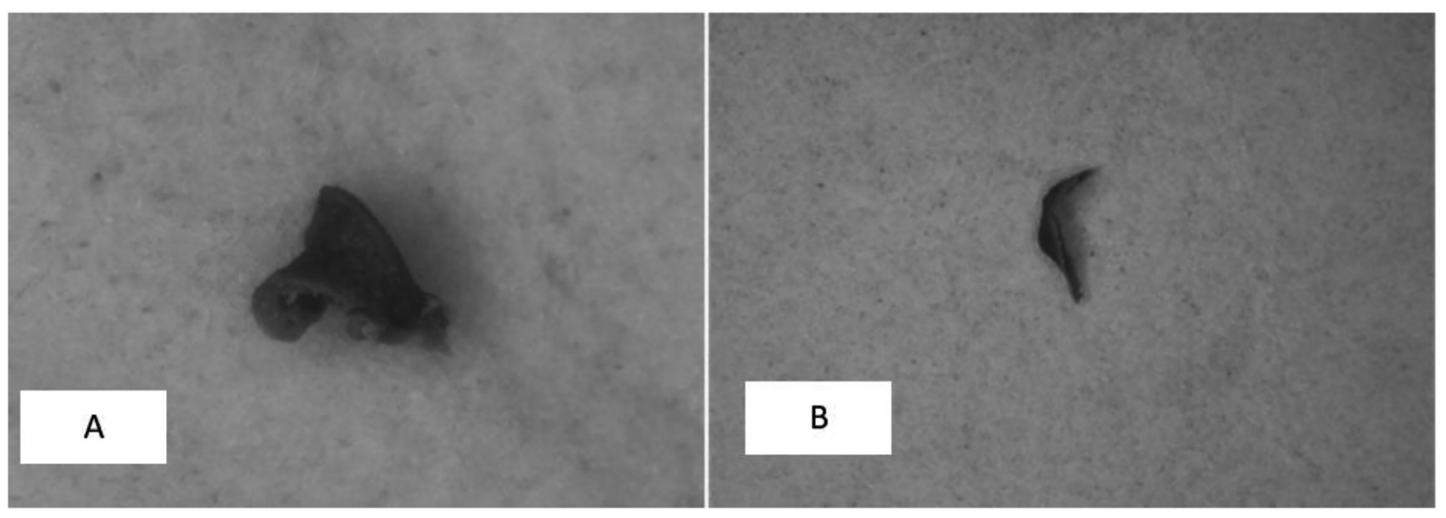

Figure 2: (A) core; (B) fragment (images viewed under 10× eyepiece lens, $1 \times$ objective lens, and zoomed $8 \times$ for a total magnification of $80 \times$ ).

stoppers, the needle entry points are obviously larger and torn when fragmentation occurred compared to when it did not.

There is an obvious difference in performance between the needles procured from Manufacturer $\mathrm{A}$ and from Manufacturer B, as evidenced by the increased number of total cores and fragments during penetration 1 while using the $20 \mathrm{G}$ needles procured from Manufacturer B. Total cores and fragments increased from none for $22 \mathrm{G}$ needles (all bevel positions) and 2 for $18 \mathrm{G}$ needles (bevel down) to 6,17 , and 9 for $20 \mathrm{G}$ needles (bevel up, down, and side, respectively). Microscopic examination of the $20 \mathrm{G}$ needle tips after penetration, as shown in Figure 4, revealed the needle tips on the $20 \mathrm{G}$ needles from Manufacturer B were prone to bending and blunting. However, when examined prior to penetration, needle tips across all manufacturers, as shown in Figure 5, appeared comparable. The bending observed in the needle tips provided by Manufacturer B occurred for $46 \%$ of the replicates, but was never observed in the $18 \mathrm{G}$ and $22 \mathrm{G}$ needles provided by Manufacturer $\mathrm{A}$. Blunt needles have been shown to result in higher fragmentation incidence [1]. Wani et al. have demonstrated that blunt plastic cannulae fragmented $40.2 \%$ of vials whereas sharp beveled needles only fragmented 4.8\% [28]. Meanwhile Eskander et al. observed their two highest incidences of fragmentation in blunt plastic cannulae $(9.9 \%)$ followed by steel blunt fill needles (1.7\%), demonstrating that the bluntness issue is not fully attributable to differences between plastic and steel performance [12]. This difference in performance for needles procured from different manufacturers introduces an unbalanced covariant of a categorical nature. Its inclusion into the statistical 
Table 3: Number of cores and fragments generated.

\begin{tabular}{|c|c|c|c|c|c|c|c|}
\hline \multicolumn{8}{|c|}{ Total cores and fragments per test parameter } \\
\hline & \multicolumn{3}{|c|}{ Penetration 1} & \multicolumn{3}{|c|}{ Penetration 2} & \\
\hline & Bevel up & Bevel down & Bevel side & Bevel up & Bevel down & Bevel side & \\
\hline 18G Needle & 0 & 2 & 0 & 0 & 44 & 2 & Manufacturer $\mathrm{A}$ \\
\hline 20G Needle & 6 & 17 & 9 & 0 & 49 & 0 & Manufacturer B \\
\hline 22G Needle & 0 & 0 & 0 & 0 & 24 & 0 & Manufacturer A \\
\hline \multicolumn{8}{|c|}{ Cores generated per test parameter } \\
\hline 18G Needle & 0 & 1 & 0 & 0 & 40 & 1 & Manufacturer A \\
\hline 20G Needle & 0 & 7 & 7 & 0 & 39 & 0 & Manufacturer B \\
\hline 22G Needle & 0 & 0 & 0 & 0 & 7 & 0 & Manufacturer A \\
\hline \multicolumn{8}{|c|}{ Fragments generated per test parameter } \\
\hline 18G Needle & 0 & 1 & 0 & 0 & 4 & 1 & Manufacturer A \\
\hline 20G Needle & 6 & 10 & 2 & 0 & 10 & 0 & Manufacturer B \\
\hline $22 \mathrm{G}$ Needle & 0 & 0 & 0 & 0 & 17 & 0 & Manufacturer A \\
\hline
\end{tabular}

\section{A}
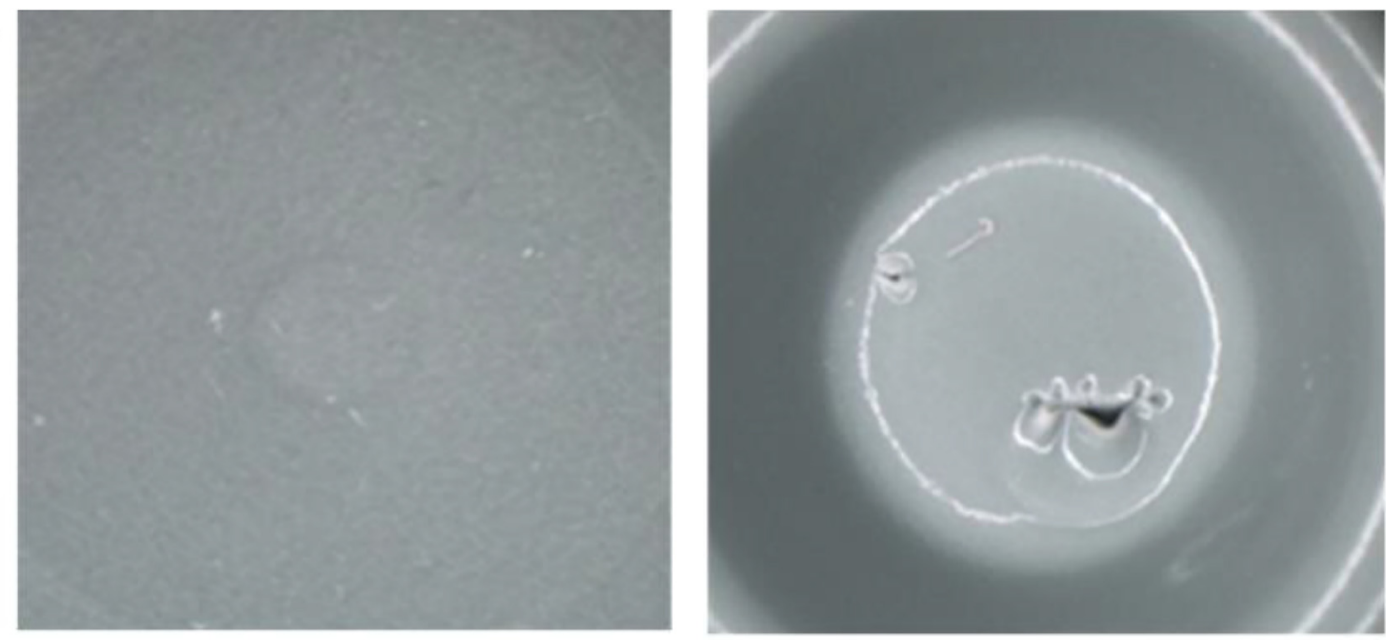

B
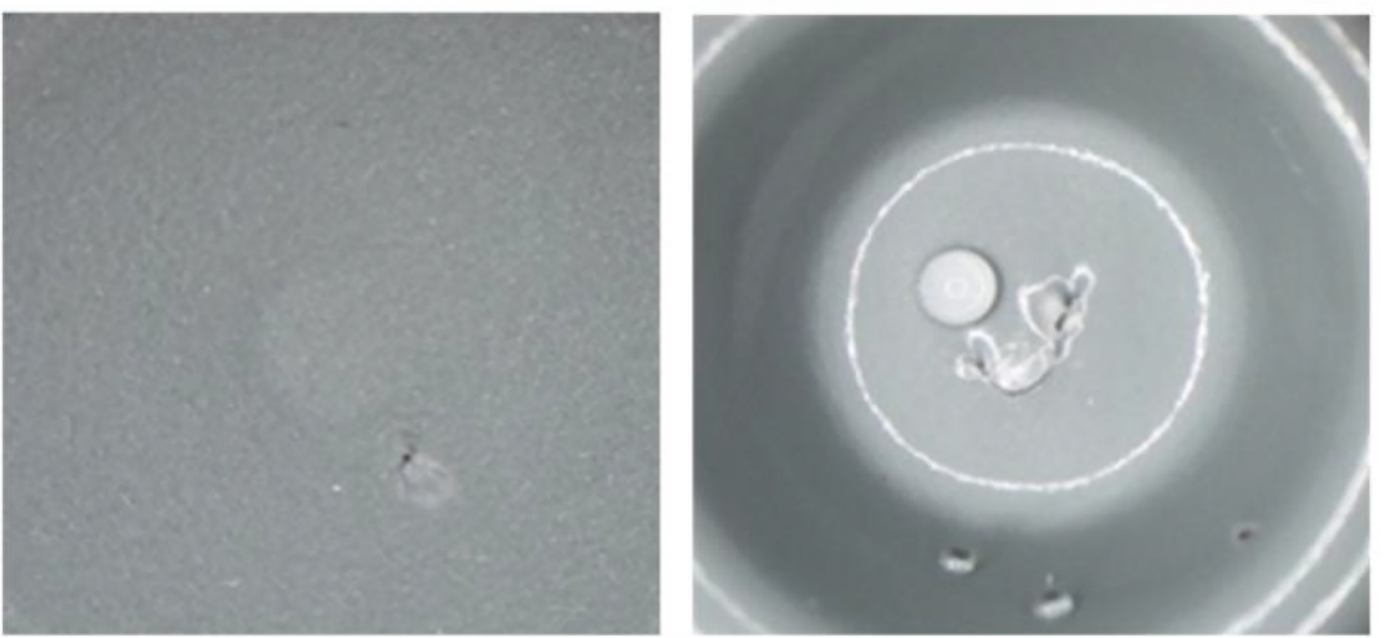

Figure 3: Images viewed under $10 \times$ eyepiece lens, $1 \times$ objective lens, and zoomed $8 \times$ for a total magnification of $80 \times(A)$ non-fragmenting stopper surface (left) and underside (right); (B) fragmenting stopper surface (left) and underside (right). 


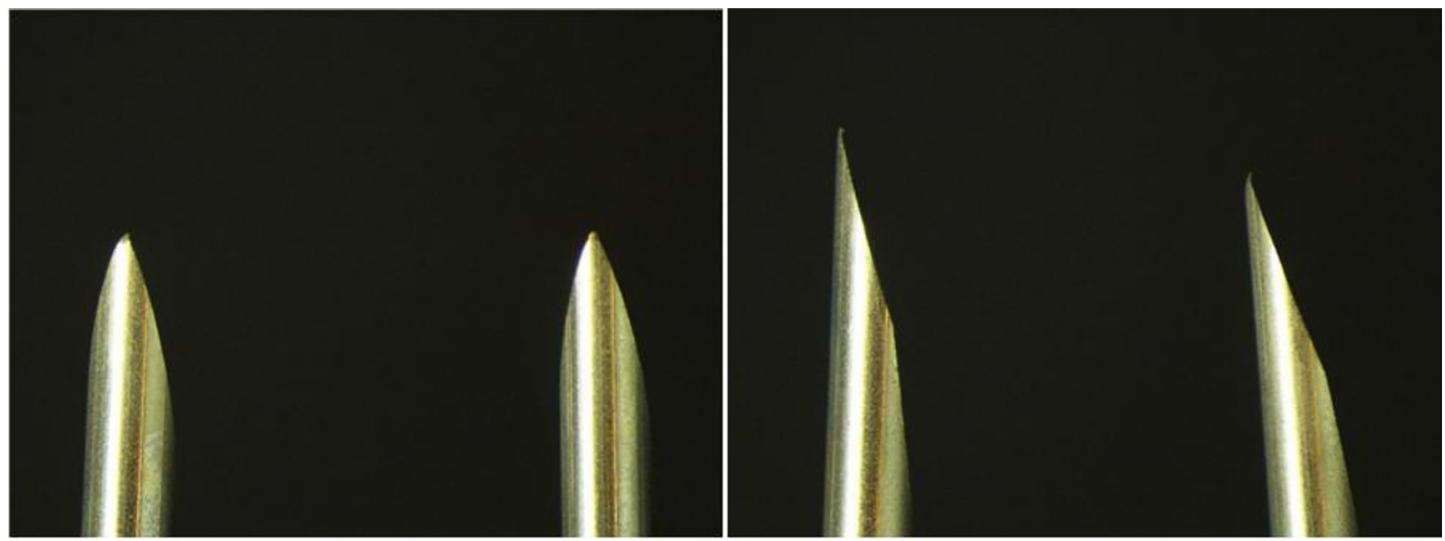

Figure 4: Examination of blunt and bent $20 \mathrm{G}$ needle tips after insertion into stopper (images viewed under $10 \times$ eyepiece lens, $1 \times$ objective lens, and zoomed $8 \times$ for a total magnification of $80 \times$ ).

analysis is therefore not straightforward and was not attempted, but it is possible that the blunting issue observed in the $20 \mathrm{G}$ needles may account for this increase in fragmentation while using the $20 \mathrm{G}$ needles with penetration 1.

Regarding the influence of bevel position on fragmentation, bevel up performed most reliably. Regardless of needle size, bevel position, and penetration method, it did not produce cores. The only particles generated were fragments at a low incidence of $6 \%$ in conjunction with the $20 \mathrm{G}$ needles using penetration method 1 . These results agree with literature sources which cite the bevel up position as the preferential orientation when penetrating stoppers [2, 3]. Meanwhile, the bevel down position produced a higher fragmentation incidence for both penetration methods, though it was notably worse for penetration method 2 as the highest incidence increased from 17 to $49 \%$ between method 1 and method 2. Moreover, it is notable that $22 \mathrm{G}$ needles did not generate cores nor fragments using penetration method 1 but fragmented at a rate of $24 \%$ using penetration method 2 with the bevel down. For penetration method 1 the bevel side position maintained a similar fragmentation rate $(6 \%)$ as the bevel up position (9\%) and both rates occurred with the $20 \mathrm{G}$ needle. For penetration method 2 the incidence was only $2 \%$ (18G needle), while bevel up did not produce cores nor fragments and bevel down performed worse with rates ranging from 24 to 49\%, depending on needle size. While controlling for needle size and penetration method, the bevel position was shown to have a statistically significant impact on both coring (Table 4, $F=20.02, D F=2, \mathrm{p}=0.000$ ) and fragmentation rates (Table 5, $F=14.62, D F=2, \mathrm{p}=0.000$ ).

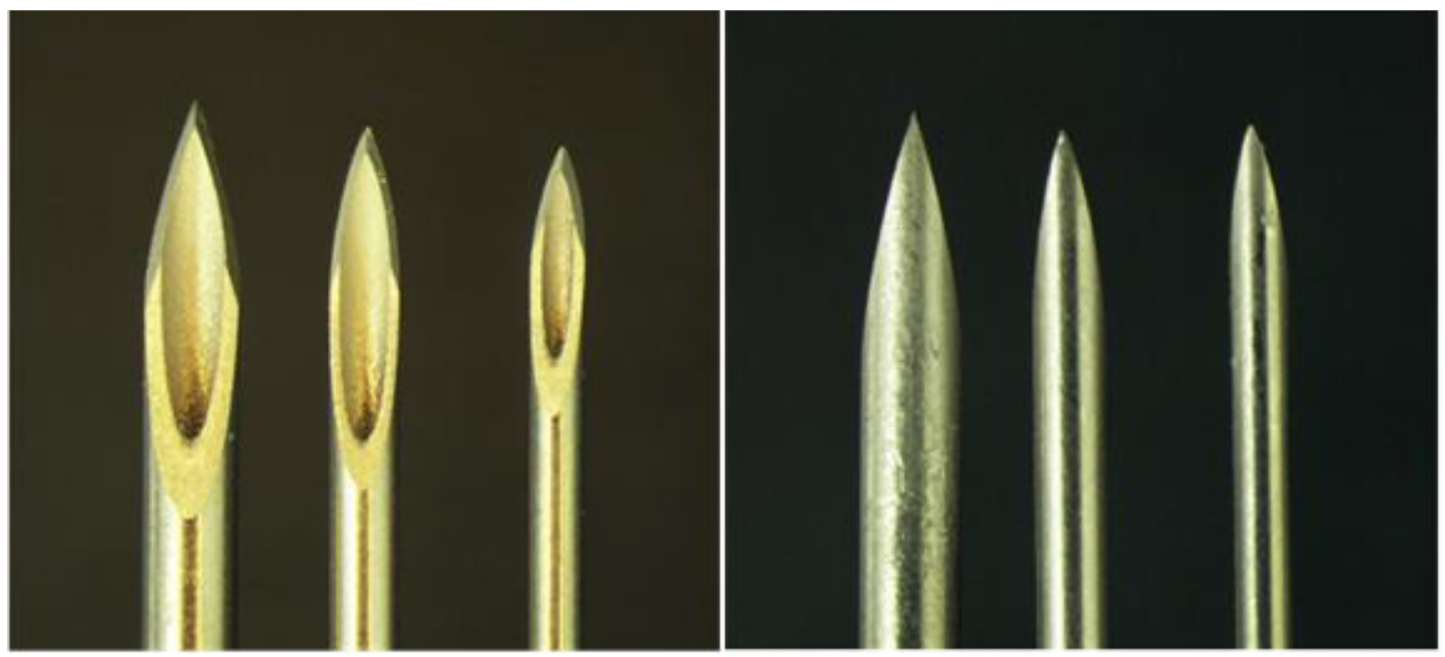

Figure 5: Examination of (left to right) 18G, 20G, and $22 \mathrm{G}$ conventional hypodermic needles (images viewed under 10× eyepiece lens, $1 \times$ objective lens, and zoomed $8 \times$ for a total magnification of $80 \times$ ). 
Regarding needle size, the smaller the needle, the smaller the fragmentation rate. This finding agrees with manufacturer recommendations to use needles smaller than $21 \mathrm{G}$ as the larger needles have larger bores which contribute to higher fragmentation rates [6, 22]. Although, fragments have been reported in vials penetrated with needles as small as 31G [13]. Moreover, with penetration method 2 the number of cores increased with needle size while the number of fragments decreased with increasing needle size. However, for penetration method 1 , while the $22 \mathrm{G}$ needles did not exhibit coring, the $18 \mathrm{G}$ needles produced only one core while the $20 \mathrm{G}$ needles produced 14 . Meanwhile the $18 \mathrm{G}$ needles produced only one fragment while the $20 \mathrm{G}$ needles produced 18 . While controlling for bevel position and penetration method, the impact of needle size was shown to be statistically significant on both coring (Table 4, $F=3.90, D F=2, \mathrm{p}=0.025$ ) and fragmentation rates (Table 5, $F=4.25, D F=2, \mathrm{p}=0.018$ ).

Regarding penetration method, while controlling for bevel position and needle size the impact of penetration method was statistically significant on coring rates (Table 4, $F=12.74, D F=1, \mathrm{p}=0.001$ ), but not on fragmentation rates (Table 5, $F=1.92, D F=1, \mathrm{p}=0.170$ ). However, it is notable that the fragmentation rate was higher using method 2. Moreover, for penetration method 1 , the incidence for cores and for fragments is comparable (8 cores vs. 11 fragments) whereas method 2 produced far more cores than fragments (86 cores vs. 31 fragments). This observation agrees with the

Table 4: General linear model - coring vs. needle gauge, needle position, and penetration method.

\begin{tabular}{|c|c|c|c|c|c|}
\hline Factor & \multicolumn{2}{|c|}{ Type } & Levels & \multicolumn{2}{|c|}{ Values } \\
\hline Needle gauge & \multicolumn{2}{|c|}{ Fixed } & 3 & \multicolumn{2}{|c|}{$18,20,22$} \\
\hline Needle position & \multicolumn{2}{|c|}{ Fixed } & 3 & \multicolumn{2}{|c|}{ Down, side, up } \\
\hline Penetration method & \multicolumn{2}{|c|}{ Fixed } & 2 & \multicolumn{2}{|c|}{1,2} \\
\hline \multicolumn{6}{|l|}{ Analysis of variance } \\
\hline Source & $D F$ & Adj. SS & Adj. MS & $F$-value & p-Value \\
\hline Needle gauge & 2 & 48.08 & 24.042 & 4.25 & 0.018 \\
\hline Needle position & 2 & 226.33 & 113.167 & 20.02 & 0.000 \\
\hline Penetration method & 1 & 72.00 & 72.000 & 12.74 & 0.001 \\
\hline Error & 66 & 373.08 & 5.653 & & \\
\hline Lack-of-fit & 12 & 326.58 & 27.215 & 31.60 & 0.000 \\
\hline Pure error & 54 & 46.50 & 0.861 & & \\
\hline Total & 71 & 719.50 & & & \\
\hline
\end{tabular}

Model summary

\begin{tabular}{lrrr}
\hline $\boldsymbol{S}$ & R-sq & R-sq (adj.) & R-sq (pred.) \\
\hline 2.37756 & $48.15 \%$ & $44.22 \%$ & $38.29 \%$
\end{tabular}

$D F=$ degrees of freedom, Adj. SS = adjusted sum of squares, Adj. $M S=$ adjusted mean squares, $R-s q=R^{2}, R-s q($ adj. $)=\operatorname{adjusted} R^{2}, R-s q$ (pred.) $=$ predicted $R^{2}$.
ANOVA results indicating that the penetration method has a significant impact on coring over fragmentation.

Finally, while using penetration method 2, operators reported requiring more pressure to pierce the stopper surface when the bevel was in the down position. It has been observed that a higher penetration force results in higher fragmentation rates [7]. In fact, this is what was observed with penetration method 2 which resulted in tearing of the stopper (Figure 6). The results of this tearing are observed as an increase in coring and fragmentation while using the bevel down position for penetration method 2. Operators also noted while performing penetration method 2 that the needles were liable to bend, snap, or slip. This handling issue poses a serious risk for needlesticks to the operator and complicates application of this penetration method. The more difficult or complicated a technique becomes, the more likely it is to be incorrectly applied or ignored. Fast and simple techniques are also preferable within emergency and high-volume or highthroughput environments such as within the operating room, emergency room, or intensive care unit where immediacy becomes paramount to successful operation.

For some services, having a low incidence of fragmentation that produced mostly larger cores may be preferential over low incidence of coring that produced small fragments because the cores are more readily observable by healthcare providers and therefore readily avoidable. Further considerations for situations like this go beyond

Table 5: General linear model - fragmentation vs. needle gauge, needle position, and penetration method.

\begin{tabular}{|c|c|c|c|c|c|}
\hline Factor & \multicolumn{2}{|c|}{ Type } & Levels & \multicolumn{2}{|c|}{ Values } \\
\hline Needle gauge & \multicolumn{2}{|c|}{ Fixed } & 3 & \multicolumn{2}{|c|}{$18,20,22$} \\
\hline Needle position & \multicolumn{2}{|c|}{ Fixed } & 3 & \multicolumn{2}{|c|}{ Down, side, up } \\
\hline Penetration method & \multicolumn{2}{|c|}{ Fixed } & 2 & \multicolumn{2}{|c|}{1,2} \\
\hline \multicolumn{6}{|l|}{ Analysis of variance } \\
\hline Source & $D F$ & Adj. SS & Adj. MS & $F$-value & p-Value \\
\hline Needle Ga. & 2 & 11.028 & 5.5139 & 3.90 & 0.025 \\
\hline Needle position & 2 & 41.361 & 20.6806 & 14.62 & 0.000 \\
\hline Penetration method & 1 & 2.722 & 2.7222 & 1.92 & 0.170 \\
\hline Error & 66 & 93.333 & 1.4141 & & \\
\hline Lack-of-fit & 12 & 49.333 & 4.1111 & 5.05 & 0.000 \\
\hline Pure error & 54 & 44.000 & 0.8148 & & \\
\hline Total & 71 & 148.444 & & & \\
\hline \multicolumn{6}{|l|}{ Model summary } \\
\hline $\boldsymbol{S}$ & R-sq & \multicolumn{2}{|c|}{ R-sq (adj.) } & \multicolumn{2}{|c|}{ R-sq (pred.) } \\
\hline 2.37756 & $37.13 \%$ & \multicolumn{2}{|r|}{$32.36 \%$} & \multicolumn{2}{|r|}{$25.17 \%$} \\
\hline
\end{tabular}



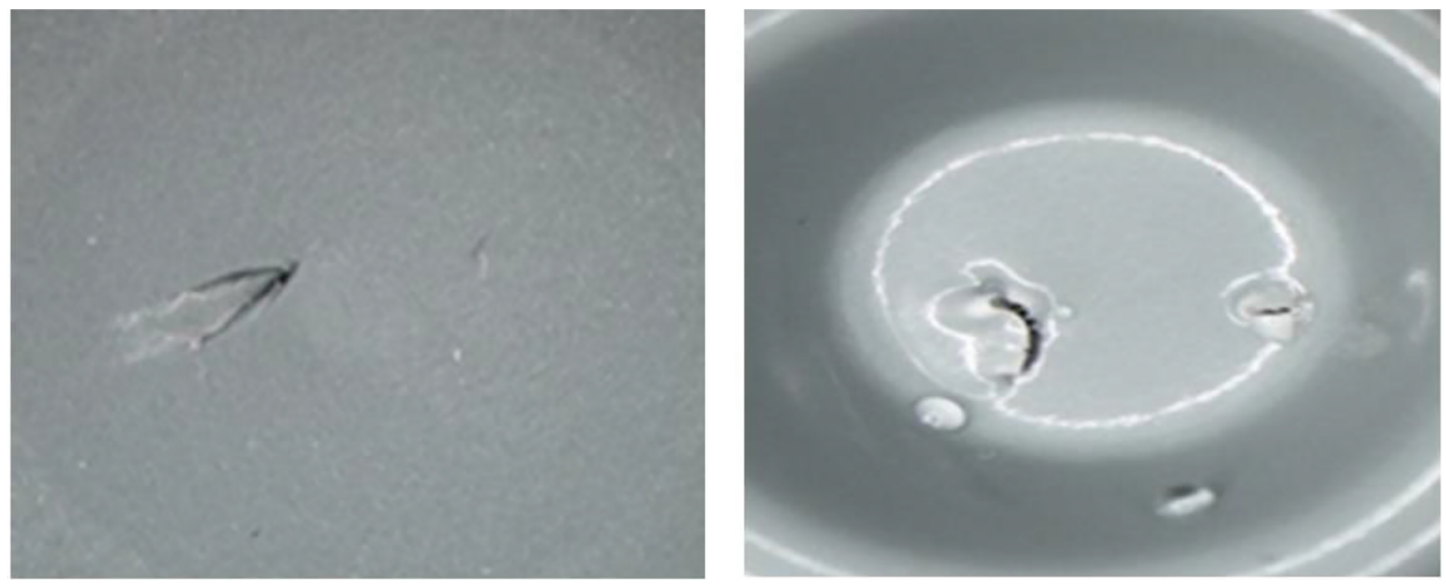

Figure 6: Tearing on stoppers penetrated using method 2 with the bevel down (images viewed under $10 \times$ eyepiece lens, $1 \times$ objective lens, and zoomed $8 \times$ for a total magnification of $80 \times$ ).

particle size as not all particles are readily observed in the clinic despite their size. Although required to examine injections before proceeding, oftentimes it can be difficult or impossible to observe particulate contaminants due to obscuration by labels, coloration of the particulate compared to that of the vial and medication, opacity of the vial and medication, size and shape of the vial, and limitations on the clinician's visual acuity $[3,11,29,30]$. These limitations demonstrate the need to de-risk medication containment and delivery solutions by the manufacturer in a way that considers clinical context and limitations. Thus, recommendations made should be conducted after specific validation questions are posed over a product's intended clinical use and supplemental functional testing performed as appropriate, as outlined in proposed general chapters $<381>$ and $<382>$ of the United States Pharmacopeia [20, 21].

\section{Conclusions}

Clearly, coring and fragmentation are impacted by multiple factors, including penetration method, needle bevel position, bevel sharpness, and needle size, all of which impact the performance of its given system significantly. This inherent variation makes the testing burden too great to validate systems for generic use across multiple devices and components, so intended use for a device should be discussed between manufacturers and healthcare providers before purchase and implementation in a clinical setting. Recommendations from manufacturers and instructions-for-use should reflect a combination of factors as they pertain to that specific penetration method (or other tested parameter) to minimize fragmentation. For example, after taking the results of this study into consideration, a device or drug provider may make the following bestpractice recommendations to a customer:

- Utilize penetration method 1 with needles $22 \mathrm{G}$ or smaller, or with needles $18 \mathrm{G}$ or smaller and the bevel facing up or to the side.

- Due to increased risk for needle-stick injury, penetration method 2 should be avoided.

Ultimately, incorporation of sterile compounding and drug withdrawal and injection protocols, as well as any other standard methods required by a practitioner's local regulations, in conjunction with the best practices outlined by a manufacturer should dictate clinical action. To adhere fully to these regulations, it is also best to purchase equipment that has been validated following appropriate and relevant international standard methods by accredited analytical laboratory services. The elastomeric closure's suitability should be based on the intended application by the end user in conjunction with standard functional testing. Any confusion or concern regarding fragmentation should be discussed with the chosen manufacturer's technical support team and fragmentation mitigation options, such as the incorporation of vial adapters and filter needles, should also be considered.

Research funding: None declared.

Author contributions: All authors have accepted responsibility for the entire content of this manuscript and approved its submission.

Competing interests: Authors state no conflict of interest. Informed consent: Not applicable.

Ethical approval: Not applicable. 


\section{References}

1. Chennell P, Bourdeaux D, Citerne Q, Arnaud C, Cosserant S, Boiko-Alaux V, et al. Rubber coring of injectable medication vial stoppers: an evaluation of causal factors. Pharm Technol Hosp Pharm 2016;1:165-74.

2. Kordi R, White BF, Kennedy DJ. Possibility and risk of medication vial coring in interventional spine procedures. PM\&R 2017;9: 289-93.

3. Roth JV. How to enter a medication vial without coring. Anesth Analg 2007;104:1615.

4. Innovation Compounding. Proper aseptic technique to prevent coring of sterile vials; 2020. Available from: https:// innovationcompounding.com/tutorial-coring/.

5. Min BJ. The West Blog: West Pharmaceutical Services, Inc. Coring and fragmentation of a rubber stopper; 2016. Available from: https://www.westpharma.com/en/blog/2016/January/coringand-fragmentation-of-a-rubber-stopper.

6. Patel J, Smith CC. Vial coring. Pain Med 2019;20:1841-2.

7. The West Blog: West Pharmaceutical Services, Inc. Causes of coring and fragmentation in the field; 2014. Available from: https://www.westpharma.com/blog/2014/June/Causes-ofCoring-and-Fragmentation-in-the-Field.

8. Ponto JA. Self-sealing capacity of vial stoppers after multiple needle punctures. J Am Pharm Assoc 2013;53:58-60.

9. Beer K. Potential foreign body emboli associated with botulinum toxin A injections. J Drugs Dermatol 2007;6:220-1.

10. Stein HA, Vu BL. Coring: a potential problem in eye surgery. J Cataract Refract Surg 1994;20:169-71.

11. Riess ML, Strong T. Near-embolization of a rubber core from a propofol vial. Anesth Analg 2008;106:1020-1.

12. Eskander J, Cotte J, Glenn E, Friedman S, Rosinia F. The incidence of coring and fragmentation of medication vial rubber stoppers. J Clin Anesth 2015;27:442-4.

13. Asakura T, Seino H, Nozaki S, Abe R. Occurrence of coring in insulin vials and possibility of rubber piece contamination by self-injection. Yakugaku Zasshi 2001;121:459-63.

14. Gragasin FS, Van den Heever ZA. The incidence of propofol vial coring with blunt needle use is reduced with angled puncture compared with perpendicular puncture. Anesth Analg 2015;120:954-5.

15. Besheer A, Mahler HC, Matter-Schwald A, Barrenechea SM, Vogt $M$, Chalus $P$, et al. Evaluation of different quality-relevant aspects of closed system transfer devices (CSTDS). Pharm Res (N Y) 2020; $37: 81$.

16. Sreedhara A, Zamiri C, Goswami S, Weiser S, Cram M, Christian TR, et al. Challenges of using closed system transfer devices with biological drug products: an industry perspective. J Pharm Sci 2020;109:22-9.

17. Stein HG. Glass ampules and filter needles: an example of implementing the sixth ' $r$ ' in medication administration. Medsurg Nurs 2006;15:290-4.
18. Gallelli JF, Groves MJ. USP perspectives on particle contamination of injectable products. J Parenter Sci Technol 1993;47:289-92.

19. Bukofzer S, Ayres J, Chavez A, Devera M, Miller J, Ross D, et al. Industry perspective on the medical risk of particles in injectable products. PDA J Pharm Sci Technol 2015;69:123-39.

20. General Chapters - Packaging and Distribution Expert Committee. General chapter <381> elastomeric components in injectable pharmaceutical product packaging/delivery systems. In: United States pharmacopeia national formulary (USP-NF).

Rockville: United States Pharmacopeial Convention; 2020: USP 41-NF 36.

21. General Chapters - Packaging and Distribution Expert Committee. General chapter <382> elastomeric component functional suitability in parenteral product packaging/delivery systems. In: United States pharmacopeia national formulary (USP-NF). Rockville: United States Pharmacopeial Convention; 2020: USP 41-NF 36.

22. Devices for administration of medicinal products and catheters committee (ISO/TC 84). Sterile hypodermic needles for single use - requirements and test methods. In: ISO 7864. International Organization for Standardization; 2016.

23. General Chapters - Dosage Forms Expert Committee. General chapter <788> particulate matter for injections. In: United States pharmacopeia national formulary (USP-NF). Rockville: United States Pharmacopeial Convention; 2015:USP 35-NF 30.

24. Council of Europe; European Pharmacopoeia Commission. Method 2.9.20 particulate contamination: visible particles. In: European Pharmacopoeia 6.0 (Ph Eur). Strasbourg: Council of Europe; 2015.

25. Society of the Japanese Pharmacopoeia. Section 6.06 Foreign insoluble matter test for injections. In: The Japanese Pharmacopoeia, 17th Edition (JP XVII). Tokyo: Society of Japanese Pharmacopoeia; 2016.

26. Shiroyama K, Izumi H, Kubo T. An advanced puncture technique decreases the incidence of coring during the use of $50-\mathrm{ml}$ propofol vials. J Anesth 2002;16:181-2.

27. Campagna R, Pessis E, Guerini H, Feydy A, Drapé JL. Occurrence of coring after needle insertion through a rubber stopper: study with prednisolone acetate. Eur Radiol 2013;23:424-7.

28. Wani T, Wadhwa A, Tobias JD. The incidence of coring with blunt versus sharp needles. J Clin Anesth 2014;26:152-4.

29. Shiroyama K. The incidence of "coring" during aspiration of propofol from a 50-mL vial. J Anesth 2001;15:120.

30. Sakai O, Furuse M, Nakashima N. Cut-off fragments of rubber caps of bottles of contrast material: foreign bodies in the drip infusion system. AJNR Am J Neuroradiol 1996;17:1194-5.

Supplementary Material: The online version of this article offers supplementary material (https://doi.org/10.1515/pthp-2021-0004). 